\title{
SIMULATION: THE SMALL MANUFACTURER'S UNKNOWN NEED
}

\author{
Walter J. Trybula
}

Ivy Systems, Incorporated

PO Box 258

Ivy, Virginia 22945

\begin{abstract}
There is a need to provide information to smaller manufacturers on the advantages of employing simulation tools. This paper examines the makeup of the hybrid microelectronics industry to identify the potential application of simulation. A specific example is used to illustrate the benefits of applying simulation.
\end{abstract}

\section{BACKGROUND}

The Hybrid Microelectronics industry is a small microcosm of the United States industry, in that there are a few large manufacturers and a large number, relatively speaking, of small manufacturers. Information about the total industry is difficult to obtain because there are a large number of captive manufacturers that have their output combined with their parent's electronics manufacturing total. In 1988, the non-captive, hybrid microelectronics industry consisted of about 450 independent manufacturers [ISHM 1987]. The largest had sales of approximately $\$ 100,000,000$, while the smallest was under $\$ 1,000,000$. Less than $10 \%$ of the companies had sales over $\$ 20,000,000$.

Automation efforts and planning for the larger companies is normally done on a regular basis. But what about the smaller companies? If we try to profile the typical small manufacturer, we end up with a description which shows that this "typical" company has sales of $\$ 7,000,000$, employees about 90 people (Based on $\$ 80 \mathrm{~K}$ sales per employee average.), has a pre-tax profit of less than $\$ 500,000$, and is constantly being bombarded with articles and literature about increasing their profitability by automating.

Having some $\$ 300,000$, after taxes, to work with for improving productivity and automating, the choices for investment are considerably narrowed. There are few managers, in this position, who would commit to install or develop a computer controlled system for their factory. It would take all their investment dollars and apparently not increase their output by one unit! What can they do? The obvious answer is to look at the various equipment vendors' literature and try to determine the best thing to buy to increase the production capacity of the factory. Unfortunately, that is not easy to do. In order to understand the problem, it is necessary to look at the industry and the processes used.

\section{HYBRID MICROELECTRONICS}

Hybrid Microelectronics (hybrids) is an interesting industry. From the early $1960 \mathrm{~s}$, it demise has been predicted "in the next few years". Obviously, since it is still here, there are some driving forces that keep it viable. In order to understand the requirements for automation, it is necessary to know a little about the industry and the requirements for its manufacturing processes.

Whether considering it a niche player or a evolving technology which constantly adapts to meet new market needs, hybrids fill a role in the electronics manufacturing community. Hybrids can be considered a packaging technology which falls between the integrated circuits and the printed wiring boards. The technology used is a combination of various processes which combine producing electronic components directly on the surface of the package to adding surface mount components to the previously built partial circuit. To evaluate the impact of automation on the complexities of the manufacturing process, it is necessary to know the process flow.

\section{MANUFACTURING}

The majority of hybrid devices are fabricated on some type of ceramic base or substrate. This material is typically alumina $\left(\mathrm{Al}_{2} \mathrm{O}_{3}\right)$ or beryllia $(\mathrm{BeO})$. On this base, the electrical circuit paths are produced. There are two basic methods of producing the paths: thin film plating or thick film screening. The thin film process is carried out in a vacuum chamber and involves the use of a sputtering system or an evaporation system which coats the substrate with various patterns of both conductive and insulative materials. This method of producing hybrids is used for the higher precision, lower power devices. Normally, this process is employed only at the larger manufacturers due to the high cost of equipment for entry into this market.

The other process, thick film screening, is more typical of smaller manufacturers. (Since these are the subject of this paper, the process will be explained in more detail.) The production of the circuitry, and some of the components, involve the screening of specially formulated thick-film pastes which are composed of finely divided metal, metal oxide, dielectric, or ferromagnetic material [Elshabine-Riad 1990]. The method of screening is a silk-screen type process where the first pattern is applied to the substrate and then it is dried in an oven to remove solvents. A second pattern can then be applied. Once all the conductive and insulative layers have been printed and dried, they are "fired" in a furnace at temperatures of approximately $850^{\circ} \mathrm{C}$. This process causes a binder, frit, in the thick film pastes to melt and react with the substrate and form an adhesion layer. The cooling is critical in order to prevent stresses in the fired films. Once through the firing process for the circuitry, the substrate returns to the screening process for the screening of resistors.

The production of thick film resistors is conducted in the same manner as the previous screenings. The key element, here, is that the nature of the resistive pastes is such that maintaining critical electronic characteristics, like resistor value sensitivity temperature, is a function of the temperature control of the firing furnace. Due to their sensitivity, resistive inks should be fired only once. Typically two to four different resistive values pastes are required for a circuit. This requires separate screens and careful positioning for each resistor paste.

Following the production of the circuitry including resistive and capacitive components, both thick and thin films have common type processes. After passing inspection, the circuits proceed to resistor and capacitor trimming. This is an adjustment process for changing the values of the screened components to meet the tolerance required for the final device. Normally, this adjustment process is performed by an abrasive trimmer or a laser trimmer. The equipment measures the value of the circuit 
element under test, removes a small portion of the element by sandblasting or vaporizing, dependent on the equipment, and retest the value of the element. This procedure continues until the value of the element is within the desired tolerance.

Once all circuit elements have been properly adjusted, the device moves into the assembly area. The procedure in assembly is basically to exactly position an adhesive on the circuit, place the discrete components on the circuit, and cure/bake/reflow the circuit to mechanically and electrically attach the components to the substrate. Obviously, there are a significant amount of detail that could be added, but are not included here because, it is not pertinent to the example presented later. After assembly, the device may receive an interim test, then will receive some final packaging, and finally goes to final test. Now the device can be shipped.

\section{AUTOMATION EFFORTS}

In any consideration of automation, it is necessary to obtain values which describe the operation of any piece of equipment. There are also many other things that must be established, i.e., hours worked per week, and test yields. Manufacturing parametrics are the numbers that define the capabilities of the facility under question. These numbers describe equipment capacity, people availability, equipment utilization, effort effectiveness, and product definition [Trybula 1990a].

\subsection{Equipment Capacity}

Of all the measurements available, the number that is most easily obtained is the equipment capacity. Manufacturer's of equipment state the speed of the equipment in devices per hour, wires per hour, passes per minute, or some similar term that provides the most favorable light for their products. In addition, there is normally a maximum and/or minimum specification or range of variables for the equipment, e.g., die sizes larger than 0.020 in. or up to a 6 in. by 6 in. substrate. This can be very misleading to the inexperienced user. It would be a very poor assumption to use both the maximum/minimum numbers along with the stated speed. In the majority of cases, the limits tend to be mutually exclusive, but they are not stated that way.

The development of throughput calculations (capacity over time) must include setup and changeover time in addition to the rate of the equipment. Throughput is defined as the actual product volume that can be reasonably expected using the anticipated equipment with established product volumes and mix. Setup time is the time to make changes when moving form one product to a similar product, e.g., changing irom a $1 \mathrm{~K}$ resistor screen to a $10 \mathrm{~K}$ screen for the same product. The times required are generally short, i.e., less than 5 minutes. Changeover is the activity that is performed when there are significant differences between the previous and the following products. In many situations, changeovers are treated as setup with extended times. Examples of this are different substrate sizes in screen printing or different methods of die attach - eutectic vs epoxy. The time involved for changeover is usually on the order of 15 to 30 minutes in hybrids, but can be significantly longer for special situations. The extra time may be required for the removal of a tooling fixture and the insertion and evaluation of a new fixture.

\subsection{The Problem}

The calculation of equipment capacity must include all these factors. For the purpose of this paper, let's consider the printing and firing operation of a small thick film hybrid manufacturer who has only one thick film printer.

If we proceed through an evaluation of capacity, we can get a idea of the problems associated with calculating actual capacities. Suppose that the question is raised, "Does the equipment have the capability of producing 300 substrates in 4 hours?" It is known that the screen printer has a capacity rating of 1,800 passes per hour. For this example, the particular device has only one conductor layer, one dielectric layer, and four resistor layers. The cycle through the drying oven is 20 minutes and the firing time is 60 minutes. The substrates in question will take priority and be processed first is there is a conflict with other products.

If the person doing the calculations has a reasonable working knowledge of his operation, he/she could calculate that there are 60 minute firing cycles and 6 print and dry cycles. In order to print 300 devices with 6 different screens, a total of 1,800 screening passes must be made - or one hour of screening time. Considering the two firing passes, a total of 3 hours out of the required 4 are used. But, the drying time has not been considered. Unfortunately, straight calculations of drying time are not accurate because the first unit printed is two thirds of the way through the drying process when the last piece is screened. So our small manufacturer might decide to tell his customer the he/she can commit to a delivery schedule based on the 4 hour print cycle.

This is a simplification of the process of developing the timing, but it will make a point. Even in a small company, the person developing the proposal or responding to a customer's call is not the hybrid engineer. In fast response situation, the process people likely will not be consulted. What the small manufacturer needs is a method of quickly simulating the process to determine the facts. As you might have guessed, the manufacturer in the example will not be able to make the scheduled delivery. What the manufacturer needs is a tool or tools that help in the decision making process.

\section{SIMULATION}

In order to determine the actual situation, there is a need for an accurate evaluation of the process interactions and the resulting production rates. There are a couple different methods available, but simulation will work best for reasons described below.

\subsection{Dynamic Simulation}

An obvious choice for accurate evaluation of the capacity of existing and proposed equipment or processes is dynamic simulation. An earlier paper [Trybula 1985], discussed the application of simulation to hybrid automation. It addressed the development of a business strategy to increase capacity by standardizing and reducing setup times, while increasing effective screening times by using multiple images per substrate. The recommendations were that automation would be significantly assisted by applying a series of standardized substrates to handle all products and reduce the fixture changes. Double the volume was achieved with only a minor investment. This paper looks at the value of simulation on the process of screening.

A model for the Screening area was developed using SIMAN from Systems Modeling Corporation. In developing the model, the screening area was treated as a station. Within this station, the printer was treated as a simple delay. A global variable was employed to establish the product type and determine the need for "changeovers". Another global variable was employed to establish the particular screening level of the product. Since the order in the queue was FIFO and due to the drying time, there was not a problem with conflict between the same product with different screening levels. The drying oven was part of this station and was treated as a constant delay. Upon exiting the drying oven, a branch statement determined whether to send the product back for more screening or on to firing. Product transfer times were set to zero.

The firing furnace was the second station. It was a simple delay with a branch at the end of the delay. This branch either sent the product for more screening or to exit the system. The characteristics of the process were 20 minutes for changeover, 10 minutes for setup of different screens, a print rate of 1800 per hour, 10 minutes drying time, and 60 minutes firing time. The 
circuit in question is the one previously described in this paper. Using the philosophy of sending product to start immediately after drying or firing gave result of 289 minutes total cycle time. If the product were grouped before being sent on to the next processing step, the maximum cycle time was 340 minutes. This latter scenario is actually more typical of the operation of a thick film hybrid facility.

\subsection{Static Simulation}

In addition to the dynamic simulation, a static simulation product, Plan_IT, was employed for a quick analysis. This is a new and promising method for facility evaluations. It is a combination of queuing theory with inputs from Operations Research that results in answers that approach dynamic simulation's answers.

The input requirements are descriptive of the overall operation. Data is entered regarding the facility requirements: hours per shift, shifts per day, non-working hours per shift, days per week, and weeks per year. The information needed to perform this evaluation is obtained through a series of questions and answers [Trybula 1990b].

The equipment and/or station information includes the weekly setup and preparation for each item, the personnel required for it to operate, any item specific overtime, and equipment failure information. An interesting feature is that MTBF and MTTR information is included in order to determine possible equipment failure and its effect on the facility.

The product information required includes the volume, the lot size, whether it is an established, prototype, or new product (for learning curve factors). The learning curve factor is adjustable but defaults to 0.9 , and is only used for prototype or new product categories.

The final set of information required is the product routing for each of the products. This includes the setup time per lot at each station, the run time per lot, and the yields. Failures and scrap product are tracked separately to calculate the total impact of the product quantity of the facility.

Once the information is entered, the calculations are extremely fast (seconds) and the results are available. The advantage of the static simulation is that many evaluations can be performed quickly. Changing lot size will change run times, to reflect lot size change, and give new cycle times and WIP levels. The availability of utilization graphs highlights the stations that have high utilizations and may be causing bottlenecks. Viewing quantities of lots processed at various stations emphasizes the key problem areas. Comparing these results with station cycle time can further identify the areas most in need of attention in the facility.

The same process steps as before were modeled using identical times. The results of the Plan_IT evaluation indicated a cycle time of 4.51 hours, or 271 minutes. The dynamic simulation was modeled, debugged, and run in less than four hours. The total modeling and running time for Plan IT was less than ten minutes for this elementary example. No debugging was required. While static simulation can not account for all the interactions that occur in this type of operating environment, the results are within $4 \%$ of the dynamic simulation results.

\section{EXPLANATION}

In order to understand the differences between the quick calculations and the simulation, let's examine what happens in the process. The order comes to the screening area with a rush priority. (Time elapsed is 00:00.) The screen printer is reset for the substrate size, the screens are taken out of storage, and a test print is run. This is the changeover mentioned earlier (00:20). The screening of conductors starts and continues for 10 minutes (300/1800 hour). (00:30) The first substrates come out of the drying oven 15 minutes after entering or 15 minutes after the start of screening $(\mathbf{0 0 : 3 5})$. The dielectric screen is now setup and QC approves the first samples (00:45). The screening is completed in 10 minutes (00:55) and the substrates are starting to come through the drying oven 5 minutes later (01:00). The substrates are now placed into the firing furnace for the 60 minute processing. The first substrates exit the furnace and are ready for more processing (02:00) [Trybula 1990a].

There are now four resistor screening passes which have a minimum time of 25 minutes for each pass. The screening of the 4 layers takes 100 minutes $(\mathbf{0 3 : 4 0})$. The addition of the final firing time adds another 60 minutes (04:40). [280 minutes] Actually, the processing of the substrates would not have begun immediately after the first substrate came out of the oven or furnace but probably after the entire group was available for processing. This would add another 60 minutes to the process (05:40). [340 minutes]

There are two points to be made by this example. The first is that other parts of the process can add additional time to the process due to the need to wait for product. The second, and more important, is that equipment capacity alone does not account for processing changes. The example used 300 substrates with 6 screening passes or a total of 1,800 passes. The equipment was rated at 1,800 passes per hour; but, the actual time that the equipment was in use on this job was 130 minutes. This indicates that the equipment was processing at a rate of 831 passes per hour, a derating factor of $46 \%$ !

\section{CONCLUSION}

The application of simulation to hybrid manufacturing would enable the manufacturer to more accurately evaluate capacity. The example described shows how a simple time estimate for job processing can be off by almost $50 \%$ due to inaccurate estimates. The problem remaining is how to convince the small manufacturer that the addition of simulation will be a highly beneficial tool for the operation. While most small manufacturers do not normally employ simulation specialists, there is a need for them to use these tools. Possible, the manufacturers of simulation languages could develop a series of industry specific, but still generic, simulation models which could be easily modified for any particular company. Until there are these tools, the best hope for the small manufacturer is to use static simulation tools like Plan_IT.

\section{REFERENCES}

Elshabine-Riad, A. and F.W. Stephenson (1990), "Hybrid Microelectronics: Old Hat for New Times?," IEEE Circuits \& Devices, p. 45.

Private communications (1987), With international society for hybrid microelectronics personnel.

Trybula, W.J. (1990a), "Automation," International Society For Hybrid Microelectronics Teaching Module, to be published by ISHM

Trybula, W.J. (1990b), "Static Simulation: A New Method of Automation Analysis," In Proceedings of the IEMT '90 Symposium, IEEE/CHMT, New York, NY.

Trybula, W.J. and R.G. Ingalls (1985), "Simulation of Hybrid Automation," In Proceedings of the 1985 International Symposium on Microelectronics, ISHM, 183-188. 